\title{
Probing light higgsinos in natural SUSY from monojet signals at the LHC
}

\author{
Chengcheng Han, ${ }^{a, b}$ Archil Kobakhidze, ${ }^{c}$ Ning Liu, ${ }^{a, c}$ Aldo Saavedra, ${ }^{c}$ Lei $\mathbf{W u}^{c}$ and \\ Jin Min Yang ${ }^{b}$ \\ ${ }^{a}$ Physics Department, Henan Normal University, \\ Xinxiang 453007, China \\ ${ }^{b}$ State Key Laboratory of Theoretical Physics, Institute of Theoretical Physics, Academia Sinica, \\ Beijing 100190, China \\ ${ }^{c}$ ARC Centre of Excellence for Particle Physics at the Terascale, School of Physics, \\ The University of Sydney, \\ NSW 2006, Australia \\ E-mail: hancheng@itp.ac.cn, archilk@physics.usyd.edu.au, \\ wlln@mail.ustc.edu.cn, a.saavedra@physics.usyd.edu.au, \\ leiwu@physics.usyd.edu.au, jmyang@itp.ac.cn
}

ABSTRACT: We investigate a strategy to search for light, nearly degenerate higgsinos within the natural MSSM at the LHC. We demonstrate that the higgsino mass range $\mu$ in $100-$ $160 \mathrm{GeV}$, which is preferred by the naturalness, can be probed at $3 \sigma$ significance through the monojet search at $14 \mathrm{TeV}$ HL-LHC with $3000 \mathrm{fb}^{-1}$ luminosity. The proposed method can also probe certain region in the parameter space for the lightest neutralino with a high higgsino purity, that cannot be reached by planned direct detection experiments at XENON-1T(2017).

KEYWORDS: Supersymmetry Phenomenology

ARXIV EPRINT: 1310.4274 


\section{Contents}

1 Introduction 1

2 Calculations and discussions 2

$\begin{array}{llr}3 & \text { Conclusion } & 7\end{array}$

\section{Introduction}

One of the key theoretical motivations for low-energy supersymmetry (SUSY) is that it provides a framework for a light Higgs boson without invoking unnatural fine-tuning of theory parameters. However, recent discovery of a Standard Model (SM) Higgs-like particle with the mass around $125 \mathrm{GeV}[1,2]$, in conjunction with non-observation of supersymmetric particles, have largely excluded the most studied parameter range within the minimal supersymmetric Standard Model (MSSM), for which the naturalness criterion is satisfied. If the observed resonance is to be identified with the lightest CP-even Higgs boson of MSSM, heavy multi-TeV stops and/or large Higgs-stop trilinear soft-breaking coupling are required to achieve sufficient enhancement of the predicted Higgs mass [3-8]. Furthermore, null results on gluino searches at the LHC so far have pushed the lower limit on gluino mass above the TeV scale [9-12]. All these significantly jeopardize the naturalness of MSSM with a standard sparticle spectrum. Therefore, it is imperative to consider the possibly hidden parameters space where the theory maintains naturalness, and look for other strategies for verifying such natural SUSY models at the LHC [13-17]. In this work, we investigate the possibility of monojet signals induced by light higgsinos at $14 \mathrm{TeV}$ high-luminosity LHC(HL-LHC) as a probe of natural SUSY.

The justification for light, nearly degenerate higgsinos within the natural MSSM comes from the following consideration. In the MSSM, the minimization of the tree-level Higgs potential leads to the relation [18]:

$$
\frac{M_{Z}^{2}}{2}=-\mu^{2}+\frac{m_{H_{d}}^{2}-m_{H_{u}}^{2} \tan ^{2} \beta}{\tan ^{2} \beta-1} \simeq-\mu^{2}-m_{H_{u}}^{2}
$$

where $m_{H_{d}}^{2}$ and $m_{H_{u}}^{2}$ represent the weak scale soft SUSY breaking masses of the Higgs fields, and $\mu$ is the higgsino mass parameter. A moderate/large $\tan \beta \gtrsim 10$ is assumed in the last approximate equation. In order to avoid large fine-tuning in eq. (1.1), $\mu$ and $m_{H_{u}}$ must be of the order of $\sim 100-200 \mathrm{GeV}$, which implies light higgsinos. At the same time, the electroweak gaugino mass parameters $M_{1,2}$ are preferred to be of the similar order as the heavy gluino mass parameter $M_{3}$ and large Higgs-stop trilinear coupling $A_{t}$ is 

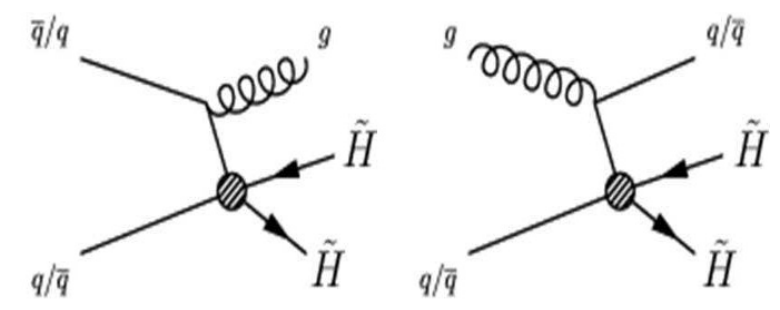

Figure 1. Feynman diagrams depicting monojet production in the natural MSSM at the LHC.

needed [19-22]. Hence, generically we have $\mu \ll M_{1,2}$ and the mass splittings between the lightest chargino and the lightest two neutralinos at leading order are determined by [27]

$$
\begin{aligned}
m_{\tilde{\chi}_{1}^{ \pm}}-m_{\tilde{\chi}_{1}^{0}}= & \frac{M_{W}^{2}}{2 M_{2}}\left(1-\sin 2 \beta-\frac{2 \mu}{M_{2}}\right) \\
& +\frac{M_{W}^{2}}{2 M_{1}} \tan ^{2} \theta_{W}(1+\sin 2 \beta), \\
m_{\tilde{\chi}_{2}^{0}}-m_{\tilde{\chi}_{1}^{0}}= & \frac{M_{W}^{2}}{2 M_{2}}\left(1-\sin 2 \beta+\frac{2 \mu}{M_{2}}\right) \\
& +\frac{M_{W}^{2}}{2 M_{1}} \tan ^{2} \theta_{W}(1-\sin 2 \beta) .
\end{aligned}
$$

This in turn implies that light electroweak gauginos in the natural MSSM are nearly degenerate higgsino-like states with a mass differences of about $3-10 \mathrm{GeV}$ (for $M_{1}=M_{2} \sim$ $0.5-2 \mathrm{TeV}$ ). Therefore, a direct search for light higgsinos may serve as a sensitive probe of the natural MSSM.

For such light higgsinos the electroweak production rates for $Z \rightarrow \tilde{\chi}_{1}^{0} \tilde{\chi}_{1}^{0}$ and $Z \rightarrow \tilde{\chi}_{2}^{0} \tilde{\chi}_{2}^{0}$ are suppressed, while the production rates for $Z / \gamma^{*} \rightarrow \tilde{\chi}_{1}^{+} \tilde{\chi}_{1}^{-}, Z \rightarrow \tilde{\chi}_{1}^{0} \tilde{\chi}_{2}^{0}, W^{ \pm} \rightarrow \tilde{\chi}_{1}^{ \pm} \tilde{\chi}_{1}^{0}$ and $W^{ \pm} \rightarrow \tilde{\chi}_{1}^{ \pm} \tilde{\chi}_{2}^{0}$ are expected to be reasonably large, reaching pb-level at the LHC. However, since the light higgsinos are nearly degenerate, the products of their subsequent decays, $\tilde{\chi}_{1}^{ \pm} \rightarrow W^{ \pm *} \tilde{\chi}_{1}^{0}$ and $\tilde{\chi}_{2}^{0} \rightarrow Z^{*} \tilde{\chi}_{1}^{0}$, will carry small energies and, hence, the currently adopted search strategy for electroweak gauginos through their direct pair production is not applicable to this case $[23,24]$. Recently, a new search channel based on the wino pair production with a same-sign diboson plus missing transverse energy $\left(\mathbb{E}_{T}\right)$ final state has been proposed for the $14 \mathrm{TeV}$ LHC in [25]. Also, it has been pointed out that for $\left(m_{\tilde{\chi}_{1}^{ \pm}}-m_{\tilde{\chi}_{1}^{0}}\right) \lesssim 1 \mathrm{GeV}$ the wino may have a long life-time and such long-lived charged particle is already excluded by the LHC data [26].

\section{Calculations and discussions}

We study the detection of the light higgsinos via monojet searches at the LHC in the following processes (see figure 1 for the corresponding Feynman diagrams):

$$
p p \rightarrow \tilde{\chi}_{1}^{ \pm} \tilde{\chi}_{1}^{\mp} j, \quad \tilde{\chi}_{1}^{0} \tilde{\chi}_{2}^{0} j, \quad \tilde{\chi}_{1}^{ \pm} \tilde{\chi}_{1,2}^{0} j .
$$

In these processes a hard jet radiated from initial partons recoils against the invisible missing transverse energy from soft decay productes and this can be used as a handle to tag the higgsino pair production. Because of the small mass splitting $(\Delta m \sim 3-10 \mathrm{GeV})$ 
between $\tilde{\chi}_{1}^{ \pm}, \tilde{\chi}_{2}^{0}$ and $\tilde{\chi}_{1}^{0}$, all three channels $\left(j \tilde{\chi}_{1}^{+} \tilde{\chi}_{1}^{-}, j \tilde{\chi}_{1}^{0} \tilde{\chi}_{2}^{0}\right.$ and $\left.j \tilde{\chi}_{1}^{ \pm} \tilde{\chi}_{1,2}^{0}\right)$ share the same topology in the detector. As a result, the monojet production rates within the natural MSSM are greatly enhanced. In addition, when $\mu \ll M_{1,2}$, these processes are largely insensitive to other SUSY parameters but higgsino mass $\mu$. Therefore, we do not consider the production of stops and gluino in this paper, which contribute to the fine-tuning in more complicated and model-dependent way [19-22]. The current constraints on the mass limits of stop and gluino in natural SUSY have been discussed in [28-33]. The sleptons and first two generation squarks are irrelevant for our analysis and we assume them to be heavy.

Since the monojets have a distinctive topology of events with a singly high $p_{T}$ hadronic jets and large missing $\mathbb{E}_{T}$, their relevance to the search for the pair production of weaklyinteracting particles have been exploited at the LHC [38, 39]. The SM backgrounds to the above monojet signature are dominated by the following four processes: (i) $p p \rightarrow Z(\rightarrow$ $\nu \bar{\nu})+j$, which is the main irreducible background with the same topology as our signals; (ii) $p p \rightarrow W(\rightarrow \ell \nu)+j$, this process fakes the signal only when the charged lepton is outside the acceptance of the detector or close to the jet; (iii) $p p \rightarrow W(\rightarrow \tau \nu)+j$, this process may fake the signal since a secondary jet from hadronic tau decays tend to localize on the side of $\mathbb{E}_{T}$; (iv) $p p \rightarrow t \bar{t}$, this process may resemble the signal, but also contains extra jets and leptons. This allows to highly suppress $t \bar{t}$ background by applying a b-jet, lepton and light jet veto.

For the QCD background, the misreconstruction of the energy of a jet in the calorimeters can cause an ordinary di-jet event with large missing energy to mimic the signal. An estimation of the QCD background based on the full detector simulation can be found in [34]. By fitting the jet energy response function (JERF) using the method in [35], the authors of [36] found that the multijet background in the supersymmetric monojets analysis at $14 \mathrm{TeV} \mathrm{LHC}$ can be reduced to a negligible level by requiring a large $\mathbb{E}_{T}$ cut, such as $\mathbb{E}_{T}>200 \mathrm{GeV}$. Since other dominant backgrounds have $\mathbb{E}_{T}>200 \mathrm{GeV}$, we set $\mathbb{E}_{T}>500 \mathrm{GeV}$ as in [37], where the cuts for the monojet events are optimized for $14 \mathrm{TeV}$ LHC, thus we can safely neglect the QCD background in our calculation (the pile-up effects at $14 \mathrm{TeV}$ HL-LHC have not been considered in the work, due to lack of the exact detector configurations.). The diboson backgrounds and single top background are not considered in our calculations due to their small cross sections compared to other backgrounds.

In the calculations we assume $M_{1}=M_{2}=1 \mathrm{TeV}$ and use the Suspect [40] and SUSYHIT [41] to calculate masses, couplings and branching ratios of the relevant sparticles. The parton level signal and background events are generated with MadGraph5 [42]. We perform parton shower and fast detector simulations with PYTHIA [43] and Delphes [44]. We cluster jets using the anti- $k_{t}$ algorithm with a cone radius $\Delta R=0.7$ [45]. In order to obtain reasonable statistics, a generator level event filter was applied which imposed a partonlevel cut of $p_{T}>120 \mathrm{GeV}$ on the first leading jet for signals and $W / Z+j$ backgrounds. It should be noted that the jet veto cuts can significantly affect the QCD corrections to the backgrounds [46]. To include the QCD effects, we generate parton-level events of $Z / W+j$ with up to two jets that are matched to the parton shower using the MLM-scheme with merging scale $Q=60 \mathrm{GeV}$ [47]. Due to the $t \bar{t}$ events containing a large number of jets, we need not generate the events with the extra hard partons, which will be strongly rejected 


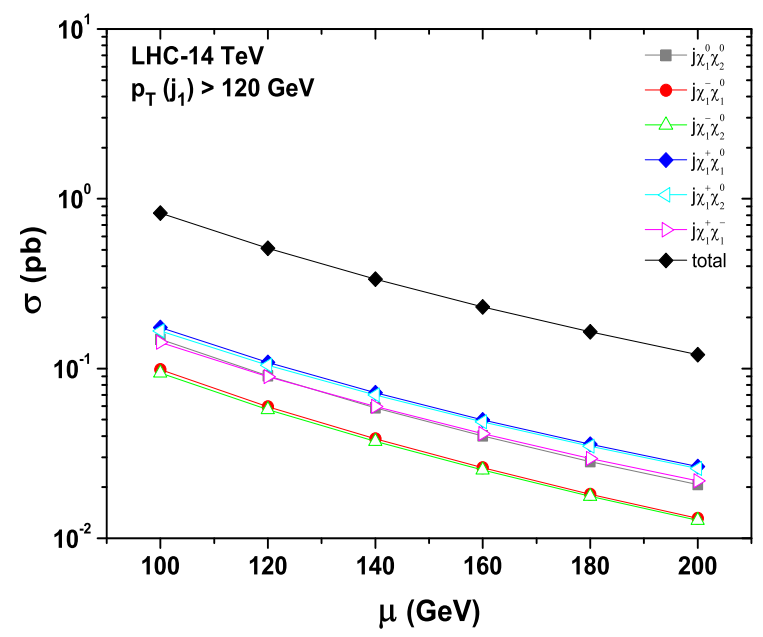

Figure 2. The parton-level cross section of monojet signals at $14 \mathrm{TeV}$ LHC.

by the jets veto [37]. Although the additional jet may come from the decays of $\tilde{\chi}_{1}^{ \pm}$or $\tilde{\chi}_{2}^{0}$, they are too soft to pass our strict $p_{T}$ cut on the leading jet adopted in the following analysis. So there is no need to generate the higgsinos pairs without additional parton in the final state. Besides, our signal simulation is exclusively based on eq. (2.1) so that double counting will not arise in our calculation.

In figure 2 , we display the cross section of $p p \rightarrow \tilde{\chi}_{1}^{ \pm} \tilde{\chi}_{1}^{\mp} j, \quad \tilde{\chi}_{1}^{0} \tilde{\chi}_{2}^{0} j, \tilde{\chi}_{1}^{ \pm} \tilde{\chi}_{1,2}^{0} j$ as a function of higgsino mass $\mu$ after requiring the parton-level cut $p_{T}\left(j_{1}\right)>120 \mathrm{GeV}$ at $14 \mathrm{TeV}$ LHC. Since $u g$ initial states have large parton distribution function, the largest contribution to the cross section of our signals comes from $\tilde{\chi}_{1}^{+} \tilde{\chi}_{1}^{0} j$. The degenerate spectrum of $\tilde{\chi}_{1}^{ \pm}$and $\tilde{\chi}_{1,2}^{0}$ implies that signals with the same initial states have approximately same cross sections. Therefore, the total production rate is amplified and can reach nearly pb-level.

In figure 3 we show the normalized distributions of a reconstructed leading jet $p_{T}\left(j_{1}\right)$ and $\mathbb{E}_{T}$ of the signals and backgrounds. From the upper panel one can see that for $p_{T}\left(j_{1}\right)>$ $200 \mathrm{GeV}$ the signals have harder $p_{T}\left(j_{1}\right)$ spectrum than the backgrounds. The greater value of $\mu$ corresponds to an increase in the average $p_{T}$ of the jet. The difference in peaks of the signals $(\sim 120 \mathrm{GeV})$ and $t \bar{t}$ background $\left(\sim m_{t} / 2\right)$ is caused by the parton-level cut $p_{T}\left(j_{1}\right)>120 \mathrm{GeV}$. From the lower panel one observes that the signals have the larger $\mathbb{E}_{T}$ than the backgrounds. Thus, a hard cut on $\mathbb{E}_{T}$ will be effective to reduce the backgrounds.

According to the above analysis, events are selected to satisfy the following criteria of monojet searches [38, 39], and the cuts for $\mathbb{E}_{T}$ and $p_{T}\left(j_{1}\right)$ are optimized for $14 \mathrm{TeV}$ LHC [37]: (i) We require large missing transverse energy $\mathbb{E}_{T}>500 \mathrm{GeV}$; (ii) The leading jet is required to have $p_{T}\left(j_{1}\right)>500 \mathrm{GeV}$ and $\left|\eta_{j_{1}}\right|<2$; events with more than two jets with $p_{T}$ above $30 \mathrm{GeV}$ in the region $|\eta|<4.5$ are rejected; (iii) We veto the second leading jet with $p_{T}\left(j_{2}\right)>100 \mathrm{GeV}$ and $\left|\eta_{j_{2}}\right|<2$; (iv) A veto on events with an identified lepton $(\ell=e, \mu, \tau)$ or $b$-jet is imposed to reduce the background of $W+j$ and $t \bar{t}$. We use the $b$-jet tagging efficiency parametrisation given in [48] and include a misidentification $10 \%$ and $1 \%$ for $c$-jets and light jets respectively. We also assume the $\tau$ tagging efficiency is $40 \%$ and include the mis-tags of QCD jets by using Delphes. 

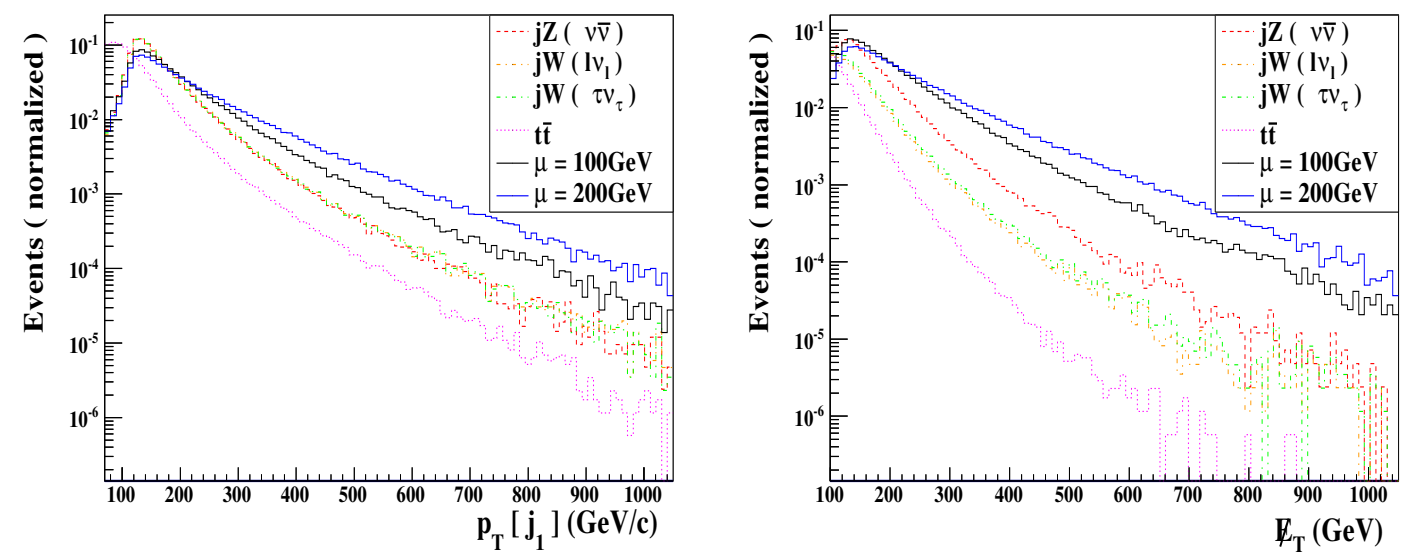

Figure 3. The normalized distributions of the reconstructed leading jet $p_{T}\left(j_{1}\right)$ and $\mathbb{E}_{T}$ of the monojet signals and backgrounds at $14 \mathrm{TeV}$ LHC.

\begin{tabular}{|c|c|c|c|c|c|c|}
\hline cut & $Z(\nu \bar{\nu})+j$ & $W\left(\ell \nu_{\ell}\right)+j$ & $W\left(\tau \nu_{\tau}\right)+j$ & $t \bar{t}$ & Signal $(\mu=100 \mathrm{GeV})$ & Signal $(\mu=200 \mathrm{GeV})$ \\
\hline$p_{T}\left(j_{1}\right)>500 \mathrm{GeV}$ & 69322 & 241740 & 119078 & 210943 & 1242 & 415 \\
\hline $\mathscr{E}_{T}>500 \mathrm{GeV}$ & 26304 & 28209 & 16513 & 2786 & 950 & 335 \\
\hline veto on $p_{T}\left(j_{2}\right)>100, p_{T}\left(j_{3}\right)>30$ & 16988 & 12194 & 7577 & 306 & 602 & 223 \\
\hline veto on $e, \mu, \tau$ & 16557 & 3963 & 3088 & 102 & 597 & 220 \\
\hline veto on $b$-jets & 16303 & 3867 & 3046 & 56 & 576 & 214 \\
\hline
\end{tabular}

Table 1. Cut flow of the signal events for $\mu=100,200 \mathrm{GeV}$ at $14 \mathrm{TeV}$ LHC with $\mathcal{L}=100 \mathrm{fb}^{-1}$. The cross section of $t \bar{t}$ is normalized to the approximately next-to-next-to-leading order value $\sigma_{t \bar{t}}=920 \mathrm{pb}[49]$.

In table 1, the resulting cut-flow for signal and background events is presented, for a centre-of-mass energy of $14 \mathrm{TeV}$ and an integrated luminosity of $100 \mathrm{fb}^{-1}$. After the cuts $P_{T}\left(j_{1}\right)>500 \mathrm{GeV}$ and $\mathbb{E}_{T}>500 \mathrm{GeV}$, the $Z+j$ and $W+j$ backgrounds are reduced by $\mathcal{O}\left(10^{-4}\right)$, while the signals only by $\mathcal{O}\left(10^{-2}\right)$. The lepton and light jet veto will suppress $W j$ backgrounds by extra two orders. For $t \bar{t}$ background, we have not included the hadronic channels due to its large jet multiplicity and small $\mathbb{E}_{T}$. We impose the third jet veto as the requirement of the ATLAS collaboration [38, 39], which is not used in the paper [37]. We also checked that our results are consistent with those obtained in ref. [50] by setting the same values of cuts and collider energy. The $Z(\nu \bar{\nu})$ process is still the dominant background after all cuts.

In figure 4 we display the dependence of the signal significance $S / \sqrt{B}$ on the higgsino mass $\mu$ at $14 \mathrm{TeV}$ HL-LHC for various luminosities, $\mathcal{L}=1000,2000,3000 \mathrm{fb}^{-1}$. The overall background $B$ including the systematic errors is calculated through the formula $B=5.3 B_{Z+j}+\sum_{i} B_{i}+\sum_{i}\left(0.1 B_{i}\right)^{2}, \quad\left(i=t \bar{t}, \mathrm{~W}\left(\rightarrow \ell \nu_{\ell}\right)+\mathrm{j}, \mathrm{W}\left(\rightarrow \tau \nu_{\tau}\right)+\mathrm{j}\right)$ in ref. [37]. With an increase of $\mu$ the significance drops fast due to the reduction in the signal cross sections. At $\mathcal{L}=3000 \mathrm{fb}^{-1}$, the range $\mu \sim 100-160 \mathrm{GeV}$, favored by the naturalness, can be probed at $3 \sigma$ significance. However, it should be mentioned that, since the realistic detector performances of the HL-LHC are still not available, we can expect our analysis can be improved by optimizing signal extraction strategies and better understanding of the backgrounds uncertainties through the dedicated analysis of the experimental collaborations at HL-LHC. 


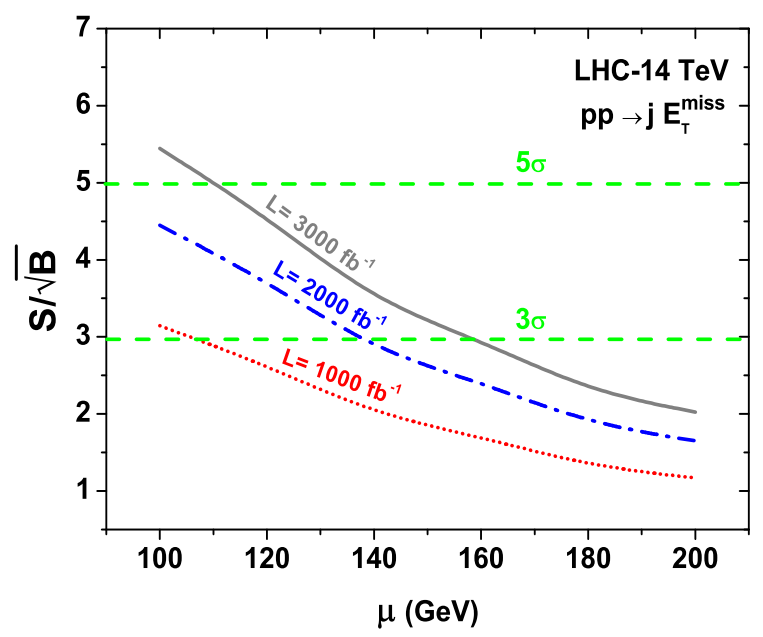

Figure 4. The dependence of significance on the higgsino mass $\mu$ at $14 \mathrm{TeV}$ HL-LHC with $\mathcal{L}=$ $1000,2000,3000 \mathrm{fb}^{-1}$.

As a complementary searches for the light higgsinos, we also investigate the probing ability of the dark matter direct detections. We computed the dark matter observables by using the package MicrOmega [51] and scan the following parameter space: $100 \mathrm{GeV} \leq$ $\mu \leq 200 \mathrm{GeV}, 0.6 \mathrm{TeV} \leq m_{\tilde{Q}_{L 3}}, m_{\tilde{t}_{R}}=m_{\tilde{b}_{R}} \leq 2 \mathrm{TeV},-3 \mathrm{TeV} \leq A_{t}=A_{b} \leq 3 \mathrm{TeV}$, $1 \leq \tan \beta \leq 60,1 \mathrm{TeV} \leq M_{1}, M_{2} \leq 2 \mathrm{TeV}$. Other irrelevant mass parameters are taken as $2 \mathrm{TeV}$. The above parameters are further constrained by: (1) Measurements of $B \rightarrow X_{s} \gamma$ and $B_{s} \rightarrow \mu^{+} \mu^{-}$processes at $2 \sigma$ level [52, 53]; (2) Higgs mass in the range 123-127 GeV [54, 55]; (3) LHC searches for $H / A \rightarrow \tau^{+} \tau^{-}$[56]; (4) Direct search results of stop/sbottom pair productions at the LHC [33]; (5) LEP data [58, 59] and (6) Electroweak precision measurements [57].

We note that, in the natural MSSM, the thermal relic density of the light higgsinolike neutralino dark matter is typically low due to the large annihilation rate in the early universe. This makes the standard thermally produced WIMP dark matter inadequate in the natural MSSM. In order to provide the required relic density, several alternative ways have been proposed [60-65], such as choosing the axion-higgsino admixture as the dark matter $[66,67]$. In this case, the spin-independent neutralino-proton scattering cross section $\sigma_{p}^{\mathrm{SI}}$ must be re-scaled by a factor $\Omega_{\tilde{\chi}_{1}^{0}} h^{2} / \Omega_{\mathrm{PL}} h^{2}[66,67]$, where $\Omega_{\mathrm{PL}} h^{2}$ is the relic density measured by Planck satellite [68]. However, it should be mentioned that, if the naturalness requirement is relaxed, the heavy higgsino-like neutralino with a mass about $1 \mathrm{TeV}$ can solely produce the correct relic density in the MSSM [69, 70]. Of course, all these analyses are performed by assuming a standard $\Lambda$ CDM model.

The results for the spin-independent higgsino-proton scattering cross section are shown in figure 5 and compared with the current limits from XENON-100, LUX [71, 72] and future reach projections of XENON-1T [73]. We also present the $3 \sigma$ probing sensitivity of the higgsino mass $\mu$ by our proposed monojet strategy at the LHC with $L=3000 \mathrm{fb}^{-1}$. From figure 5 we can see that even with the scale factor $\Omega_{\tilde{\chi}_{1}^{0}} h^{2} / \Omega_{\mathrm{PL}} h^{2}$, most of the samples can be probed by the XENON-1T(2017). Only those samples corresponding to a neutralino 


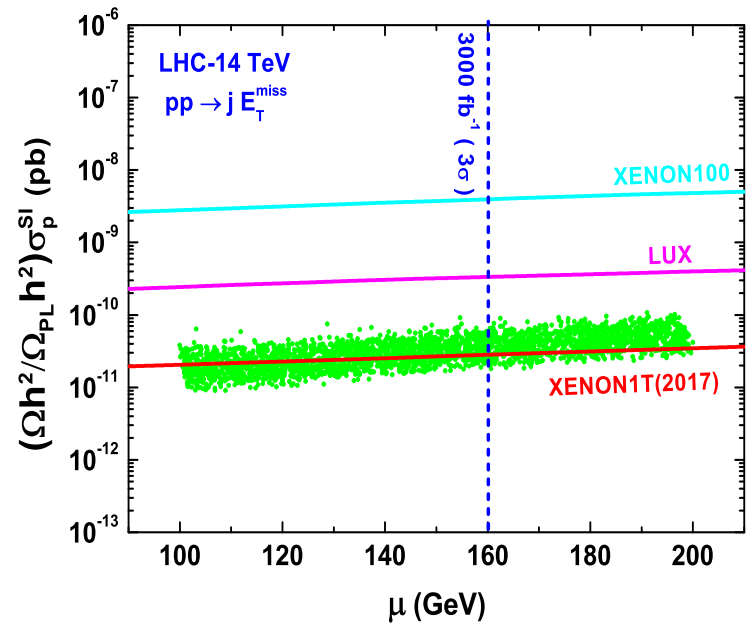

Figure 5. Scatter plot of samples survived the constraints from (1)-(6) in the text. The horizontal lines show the $90 \%$ C.L. bound from XENON100 [71], future sensitivities at LUX [72] and XENON1T [73], respectively. The vertical dashed line is the sensitivity of monojet signals at $3 \sigma$ significance at $14 \mathrm{TeV}$ LHC with $\mathcal{L}=3000 \mathrm{fb}^{-1}$.

with a high higgsino purity can not be covered by the XENON-1T(2017). In this case, our proposed monojet searches may be used to probe such a light higgsino-dominant neutralino with mass up to $\sim 160 \mathrm{GeV}$ at $14 \mathrm{TeV}$ LHC for $L=3000 \mathrm{fb}^{-1}$.

\section{Conclusion}

In this paper, we studied a strategy for searching light, nearly degenerate higgsinos in the natural MSSM. Our results showed that for $\mathcal{L}=3000 \mathrm{fb}^{-1}$, the higgsino mass range $\mu \sim 100-160 \mathrm{GeV}$ favored by the naturalness may be probed at $3 \sigma$ significance through the monojet searches at $14 \mathrm{TeV}$ LHC. Also, this method can probe certain area in the parameter space for the lightest neutralino with a high higgsino purity, that cannot be reached by planned direct detection experiments at XENON-1T(2017).

\section{Acknowledgments}

This work was supported by the Australian Research Council, by the National Natural Science Foundation of China (NNSFC) under grants No. 11275245, 10821504, 11135003 and 11305049, by Specialized Research Fund for the Doctoral Program of Higher Education under Grant No. 20134104120002 and by the Startup Foundation for Doctors of Henan Normal University under contract No. 11112.

Open Access. This article is distributed under the terms of the Creative Commons Attribution License (CC-BY 4.0), which permits any use, distribution and reproduction in any medium, provided the original author(s) and source are credited. 


\section{References}

[1] ATLAS collaboration, Combined search for the Standard Model Higgs boson using up to $4.9 \mathrm{fb}^{-1}$ of $p p$ collision data at $\sqrt{\mathrm{s}}=7 \mathrm{TeV}$ with the ATLAS detector at the LHC, Phys. Lett. B 710 (2012) 49 [arXiv:1202.1408] [INSPIRE].

[2] CMS collaboration, Combined results of searches for the standard model Higgs boson in pp collisions at $\sqrt{s}=7$ TeV, Phys. Lett. B 710 (2012) 26 [arXiv:1202.1488] [INSPIRE].

[3] M.S. Carena and H.E. Haber, Higgs boson theory and phenomenology, Prog. Part. Nucl. Phys. 50 (2003) 63 [hep-ph/0208209] [InSPIRE].

[4] A. Arbey, M. Battaglia, A. Djouadi, F. Mahmoudi and J. Quevillon, Implications of a 125 GeV Higgs for supersymmetric models, Phys. Lett. B 708 (2012) 162 [arXiv:1112.3028] [INSPIRE].

[5] M. Carena, S. Gori, N.R. Shah and C.E. Wagner, A 125 GeV SM-like Higgs in the MSSM and the $\gamma \gamma$ rate, JHEP 03 (2012) 014 [arXiv:1112.3336] [INSPIRE].

[6] J. Cao, Z. Heng, J.M. Yang and J. Zhu, Status of low energy SUSY models confronted with the LHC 125 GeV Higgs data, JHEP 10 (2012) 079 [arXiv:1207.3698] [INSPIRE].

[7] J.-J. Cao, Z.-X. Heng, J.M. Yang, Y.-M. Zhang and J.-Y. Zhu, A SM-like Higgs near $125 \mathrm{GeV}$ in low energy SUSY: a comparative study for MSSM and NMSSM, JHEP 03 (2012) 086 [arXiv:1202.5821] [INSPIRE].

[8] J. Cao, Z. Heng, D. Li and J.M. Yang, Current experimental constraints on the lightest Higgs boson mass in the constrained MSSM, Phys. Lett. B 710 (2012) 665 [arXiv:1112.4391] [INSPIRE].

[9] ATLAS collaboration, Search for new phenomena using final states with large jet multiplicities and missing transverse momentum with ATLAS in $20 \mathrm{fb}^{-1}$ of $\sqrt{\mathrm{s}}=8 \mathrm{TeV}$ proton-proton collisions, ATLAS-CONF-2013-054 (2013).

[10] ATLAS collaboration, Search for strong production of supersymmetric particles in final states with missing transverse momentum and at least three b-jets using $20.1 \mathrm{fb}^{-1}$ of pp collisions at $\sqrt{s}=8 \mathrm{TeV}$ with the ATLAS Detector., ATLAS-CONF-2013-061 (2013).

[11] CMS collaboration, Search for supersymmetry in pp collisions at $\sqrt{s}=8$ TeV in events with a single lepton, large jet multiplicity and multiple b jets, CMS-SUS-13-007 (2013).

[12] CMS collaboration, Search for supersymmetry using razor variables in events with b-jets in pp collisions at $8 \mathrm{Te}$, CMS-PAS-SUS-13-004.

[13] C. Brust, A. Katz, S. Lawrence and R. Sundrum, SUSY, the Third Generation and the LHC, JHEP 03 (2012) 103 [arXiv: 1110.6670] [INSPIRE].

[14] M. Papucci, J.T. Ruderman and A. Weiler, Natural SUSY Endures, JHEP 09 (2012) 035 [arXiv: 1110.6926] [INSPIRE].

[15] L.J. Hall, D. Pinner and J.T. Ruderman, A Natural SUSY Higgs Near 126 GeV, JHEP 04 (2012) 131 [arXiv:1112.2703] [INSPIRE].

[16] J.L. Feng and D. Sanford, A Natural 125 GeV Higgs Boson in the MSSM from Focus Point Supersymmetry with A-Terms, Phys. Rev. D 86 (2012) 055015 [arXiv:1205.2372] [INSPIRE].

[17] H. Baer, V. Barger, P. Huang, A. Mustafayev and X. Tata, Radiative natural SUSY with a 125 GeV Higgs boson, Phys. Rev. Lett. 109 (2012) 161802 [arXiv:1207.3343] [InSPIRE]. 
[18] R.L. Arnowitt and P. Nath, Loop corrections to radiative breaking of electroweak symmetry in supersymmetry, Phys. Rev. D 46 (1992) 3981 [INSPIRE].

[19] S.P. Martin, Non-universal gaugino masses from non-singlet F-terms in non-minimal unified models, Phys. Rev. D 79 (2009) 095019 [arXiv:0903.3568] [INSPIRE].

[20] J.E. Younkin and S.P. Martin, Non-universal gaugino masses, the supersymmetric little hierarchy problem and dark matter, Phys. Rev. D 85 (2012) 055028 [arXiv:1201.2989] [INSPIRE].

[21] S. Akula and P. Nath, Gluino-driven Radiative Breaking, Higgs Boson Mass, Muon g-2 and the Higgs Diphoton Decay in SUGRA Unification, Phys. Rev. D 87 (2013) 115022 [arXiv: 1304.5526] [INSPIRE].

[22] I. Gogoladze, F. Nasir and Q. Shafi, Non-Universal Gaugino Masses and Natural Supersymmetry, Int. J. Mod. Phys. A 28 (2013) 1350046 [arXiv:1212.2593] [InSPIRE].

[23] J.F. Gunion and S. Mrenna, A study of SUSY signatures at the Tevatron in models with near mass degeneracy of the lightest chargino and neutralino, Phys. Rev. D 62 (2000) 015002 [hep-ph/9906270] [INSPIRE].

[24] S. Gori, S. Jung and L.-T. Wang, Cornering electroweakinos at the LHC, arXiv:1307.5952 [INSPIRE].

[25] H. Baer et al., Same sign diboson signature from supersymmetry models with light higgsinos at the LHC, Phys. Rev. Lett. 110 (2013) 151801 [arXiv:1302.5816] [INSPIRE].

[26] N.E. Bomark, A. Kvellestad, S. Lola, P. Osland and A. Raklev, Long lived charginos in Natural SUSY?, arXiv:1310.2788 [INSPIRE].

[27] G.F. Giudice and A. Pomarol, Mass degeneracy of the Higgsinos, Phys. Lett. B 372 (1996) 253 [hep-ph/9512337] [INSPIRE].

[28] J. Cao, C. Han, L. Wu, J.M. Yang and Y. Zhang, Probing Natural SUSY from Stop Pair Production at the LHC, JHEP 11 (2012) 039 [arXiv: 1206.3865] [INSPIRE].

[29] M.L. Graesser and J. Shelton, Hunting Asymmetric Stops, Phys. Rev. Lett. 111 (2013) 121802 [arXiv:1212.4495] [INSPIRE].

[30] O. Buchmueller and J. Marrouche, Universal mass limits on gluino and third-generation squarks in the context of Natural-like SUSY spectra, arXiv:1304.2185 [INSPIRE].

[31] G.D. Kribs, A. Martin and A. Menon, Natural Supersymmetry and Implications for Higgs physics, Phys. Rev. D 88 (2013) 035025 [arXiv: 1305.1313] [INSPIRE].

[32] K. Kowalska and E.M. Sessolo, Natural MSSM after the LHC 8 TeV run, Phys. Rev. D 88 (2013) 075001 [arXiv: 1307.5790] [INSPIRE].

[33] C. Han, K.-i. Hikasa, L. Wu, J.M. Yang and Y. Zhang, Current experimental bounds on stop mass in natural SUSY, JHEP 10 (2013) 216 [arXiv:1308.5307] [INSPIRE].

[34] ATLAS collaboration, Expected Performance of the ATLAS Experiment - Detector, Trigger and Physics, arXiv:0901.0512 [INSPIRE].

[35] A.J. Barr and C. Gwenlan, The race for supersymmetry: Using $m_{T 2}$ for discovery, Phys. Rev. D 80 (2009) 074007 [arXiv:0907.2713] [INSPIRE].

[36] B.C. Allanach, S. Grab and H.E. Haber, Supersymmetric Monojets at the Large Hadron Collider, JHEP 01 (2011) 138 [Erratum ibid. 1107 (2011) 087] [Erratum ibid. 1109 (2011) 027] [arXiv: 1010.4261] [INSPIRE]. 
[37] M. Drees, M. Hanussek and J.S. Kim, Light Stop Searches at the LHC with Monojet Events, Phys. Rev. D 86 (2012) 035024 [arXiv: 1201.5714] [InSPIRE].

[38] ATLAS collaboration, Search for New Phenomena in Monojet plus Missing Transverse Momentum Final States using $10 \mathrm{fb}^{-1}$ of pp Collisions at $\sqrt{s}=8 \mathrm{TeV}$ with the ATLAS detector at the LHC, ATLAS-CONF-2012-147 (2012).

[39] CMS collaboration, Search for new physics in monojet events in pp collisions at $\sqrt{s}=8 \mathrm{TeV}$, CMS-PAS-EXO-12-048.

[40] A. Djouadi, J.-L. Kneur and G. Moultaka, SuSpect: A Fortran code for the supersymmetric and Higgs particle spectrum in the MSSM, Comput. Phys. Commun. 176 (2007) 426 [hep-ph/0211331] [INSPIRE].

[41] A. Djouadi, M. Muhlleitner and M. Spira, Decays of supersymmetric particles: The program SUSY-HIT (SUspect-SdecaY-HDECAY-InTerface), Acta Phys. Polon. B 38 (2007) 635 [hep-ph/0609292] [INSPIRE].

[42] J. Alwall, M. Herquet, F. Maltoni, O. Mattelaer and T. Stelzer, MadGraph 5: Going Beyond, JHEP 06 (2011) 128 [arXiv: 1106.0522] [INSPIRE].

[43] T. Sjöstrand, S. Mrenna and P.Z. Skands, PYTHIA 6.4 Physics and Manual, JHEP 05 (2006) 026 [hep-ph/0603175] [INSPIRE].

[44] J. de Favereau et al., DELPHES 3, A modular framework for fast simulation of a generic collider experiment, arXiv:1307.6346 [INSPIRE].

[45] M. Cacciari, G.P. Salam and G. Soyez, The anti-k(t) jet clustering algorithm, JHEP 04 (2008) 063 [arXiv: 0802.1189] [INSPIRE].

[46] A. Denner, S. Dittmaier, T. Kasprzik and A. Mück, Electroweak corrections to monojet production at the LHC, Eur. Phys. J. C 73 (2013) 2297 [arXiv:1211.5078] [InSPIRE].

[47] F. Caravaglios, M.L. Mangano, M. Moretti and R. Pittau, A new approach to multijet calculations in hadron collisions, Nucl. Phys. B 539 (1999) 215 [hep-ph/9807570] [INSPIRE].

[48] CMS collaboration, b-Jet Identification in the CMS Experiment, CMS-PAS-BTV-11-004.

[49] N. Kidonakis, The top quark rapidity distribution and forward-backward asymmetry, Phys. Rev. D 84 (2011) 011504 [arXiv: 1105.5167] [INSPIRE].

[50] B. Bhattacherjee, D. Choudhury, K. Harigaya, S. Matsumoto and M.M. Nojiri, Model Independent Analysis of Interactions between Dark Matter and Various Quarks, JHEP 04 (2013) 031 [arXiv:1212.5013] [INSPIRE].

[51] G. Bélanger et al., Indirect search for dark matter with MicrOMEGAs2.4, Comput. Phys. Commun. 182 (2011) 842 [arXiv:1004.1092] [INSPIRE].

[52] F. Mahmoudi, SuperIso v2.3: A program for calculating flavor physics observables in Supersymmetry, Comput. Phys. Commun. 180 (2009) 1579 [arXiv:0808.3144] [InSPIRE].

[53] F. Mahmoudi, SuperIso: A program for calculating the isospin asymmetry of $B \rightarrow K^{*} \gamma$ in the MSSM, Comput. Phys. Commun. 178 (2008) 745 [arXiv:0710.2067] [InSPIRE].

[54] S. Heinemeyer, W. Hollik and G. Weiglein, FeynHiggs: A program for the calculation of the masses of the neutral CP even Higgs bosons in the MSSM,

Comput. Phys. Commun. 124 (2000) 76 [hep-ph/9812320] [INSPIRE].

[55] S. Heinemeyer, W. Hollik and G. Weiglein, The masses of the neutral CP-even Higgs bosons in the MSSM: Accurate analysis at the two loop level, Eur. Phys. J. C 9 (1999) 343 [hep-ph/9812472] [INSPIRE]. 
[56] CMS collaboration, Higgs to tau tau (MSSM) (HCP), CMS-PAS-HIG-12-050.

[57] J. Cao and J.M. Yang, Anomaly of Zb $\bar{b}$ coupling revisited in MSSM and NMSSM, JHEP 12 (2008) 006 [arXiv:0810.0751] [INSPIRE].

[58] P. Bechtle, O. Brein, S. Heinemeyer, G. Weiglein and K.E. Williams, HiggsBounds 2.0.0: Confronting Neutral and Charged Higgs Sector Predictions with Exclusion Bounds from LEP and the Tevatron, Comput. Phys. Commun. 182 (2011) 2605 [arXiv:1102.1898] [INSPIRE].

[59] P. Bechtle, O. Brein, S. Heinemeyer, G. Weiglein and K.E. Williams, HiggsBounds: Confronting Arbitrary Higgs Sectors with Exclusion Bounds from LEP and the Tevatron, Comput. Phys. Commun. 181 (2010) 138 [arXiv:0811.4169] [InSPIRE].

[60] B.S. Acharya, G. Kane and E. Kuflik, String Theories with Moduli Stabilization Imply Non-Thermal Cosmological History and Particular Dark Matter, arXiv:1006.3272 [INSPIRE].

[61] T. Moroi and L. Randall, Wino cold dark matter from anomaly mediated SUSY breaking, Nucl. Phys. B 570 (2000) 455 [hep-ph/9906527] [INSPIRE].

[62] G.B. Gelmini and P. Gondolo, Neutralino with the right cold dark matter abundance in (almost) any supersymmetric model, Phys. Rev. D 74 (2006) 023510 [hep-ph/0602230] [INSPIRE].

[63] B.S. Acharya, K. Bobkov, G.L. Kane, P. Kumar and J. Shao, Explaining the Electroweak Scale and Stabilizing Moduli in M-theory, Phys. Rev. D 76 (2007) 126010 [hep-th/0701034] [INSPIRE].

[64] B.S. Acharya, K. Bobkov, G.L. Kane, J. Shao and P. Kumar, The $G_{2}-M S S M$ : An M-theory motivated model of Particle Physics, Phys. Rev. D 78 (2008) 065038 [arXiv:0801.0478] [INSPIRE].

[65] K.-Y. Choi, J.E. Kim, H.M. Lee and O. Seto, Neutralino dark matter from heavy axino decay, Phys. Rev. D 77 (2008) 123501 [arXiv:0801.0491] [InSPIRE].

[66] H. Baer, A. Lessa, S. Rajagopalan and W. Sreethawong, Mixed axion/neutralino cold dark matter in supersymmetric models, JCAP 06 (2011) 031 [arXiv:1103.5413] [INSPIRE].

[67] H. Baer, A. Lessa and W. Sreethawong, Coupled Boltzmann calculation of mixed axion/neutralino cold dark matter production in the early universe, JCAP 01 (2012) 036 [arXiv:1110.2491] [INSPIRE].

[68] Planck collaboration, P. Ade et al., Planck 2013 results. XVI. Cosmological parameters, arXiv: 1303.5076 [INSPIRE].

[69] U. Chattopadhyay and D. Roy, Higgsino dark matter in a SUGRA model with nonuniversal gaugino masses, Phys. Rev. D 68 (2003) 033010 [hep-ph/0304108] [INSPIRE].

[70] U. Chattopadhyay, D. Choudhury, M. Drees, P. Konar and D. Roy, Looking for a heavy Higgsino LSP in collider and dark matter experiments, Phys. Lett. B 632 (2006) 114 [hep-ph/0508098] [INSPIRE].

[71] XENON100 collaboration, E. Aprile et al., Dark Matter Results from 225 Live Days of XENON100 Data, Phys. Rev. Lett. 109 (2012) 181301 [arXiv:1207.5988] [InSPIRE].

[72] LUX collaboration, D. Akerib et al., The Large Underground Xenon (LUX) Experiment, Nucl. Instrum. Meth. A 704 (2013) 111 [arXiv:1211.3788] [InSPIRE].

[73] XENON1T collaboration, E. Aprile, The XENON1T Dark Matter Search Experiment, arXiv:1206.6288 [INSPIRE]. 